\title{
Thermal hysteresis of antifreeze proteins considering Fragilariopsis cylindrus
}

\author{
Bernd Kutschan ${ }^{1}$, Silke Thoms² \& Maddalena Bayer-Giraldi² \\ ${ }^{1}$ FH Münster, Corrensstr. 25, 48149 Münster, Germany \\ ${ }^{2}$ Alfred Wegener Institute, Helmholtz Centre for Polar and Marine Research, \\ Am Han-delshafen 12, 27570 Bremerhaven, Germany
}

With 9 figures and 1 table

\begin{abstract}
The diatom species Fragilariopsis cylindrus produces antifreeze proteins (AFPs) of moderate thermal hysteresis. Two concepts are often used in order to describe a thermal hysteresis, on the one hand the irreversible nucleation growth described by the Gibbs-Thomson relation and on the other hand a nonlinear adsorption kinetics of the ice-binding proteins. We refer to the Landau's phase transition theory and predict a saturation concentration of AFPs for the maximal freezing depression. The derived functional relation between temperature and AFP concentration is more complex than a simple power law as suggested by thermal hysteresis experiments. With the parameter set obtained for Fragilariopsis cylindrus the modelled curve is comparable with the often used square root law.
\end{abstract}

Keywords: antifreeze proteins, thermal hysteresis, phase transition

\section{Introduction}

Diatoms represent an important fraction of the sea-ice microbial community (Brown \& Bowman 2001), which considerably contributes to the productivity of the polar oceans. They remain trapped in the ice during sea-ice formation, and survive and thrive within a porous system of brine channels and brine pockets (Thomas \& Dieckmann 2002). The diatom Fragilariopsis cylindrus is one dominant species within seaice microbial assemblages (Bartsch 1989, Günther \& Dieckmann 2001, Lizotte 2001, Thomas \& Dieckmann 2002, Roberts et al. 2007). As an adaptation to sea-ice conditions, this species produces antifreeze-proteins (AFPs) (Bayer-Giraldi et al. 2010). These proteins have been shown to modify the microstructure of ice crystals, i.e. their 
(A)

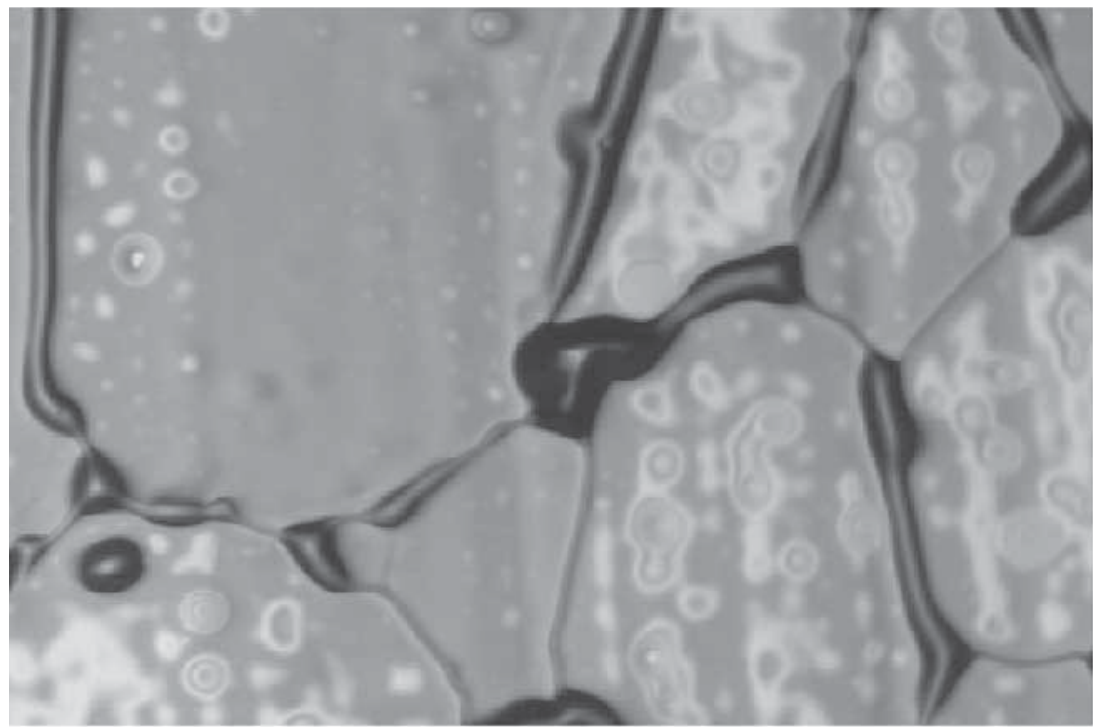

\section{$100 \mu \mathrm{m}$}

(B)

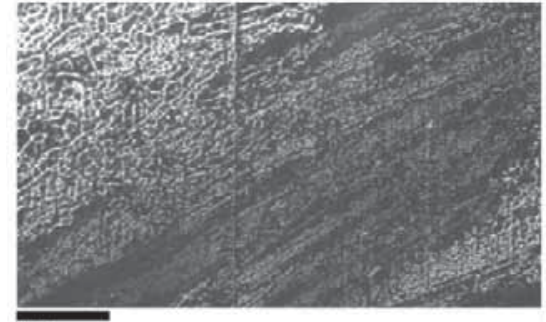

$100 \mathrm{um}$

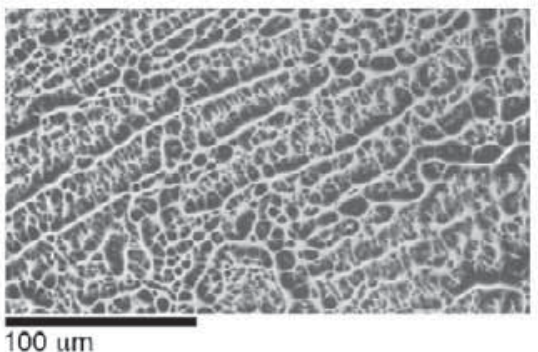

Fig. 1. Frozen samples of protein solutions observed by reflected light microscopy. (A) Negative control, frozen solution of bovine serum albumin $(0.005 \mu \mathrm{M})$, (B) Frozen solution of AFPs from F. cylindrus $(0.012 \mu \mathrm{M})$ (Reprinted with permission from Bayer-Giraldi et al. 2011).

size and shape (Bayer-Giraldi et al. 2011, Fig. 1). Diatoms presumably secrete the proteins into the brine system to modify the surface of ice, in order to maintain and shape their habitat conveniently.

Antifreeze proteins have the characteristic property of shifting the freezing point of a solution below the melting point, therefore causing a thermal hysteresis (Barrett 2001). The proteins attach to nascent ice crystals and inhibit further growth until a critical temperature, defined as freezing point, is reached (Kristiansen \& Zachariassen 2005). Ice growth is resumed at the freezing point. Antifreeze proteins are therefore also called thermal hysteresis proteins, ice-binding or ice-structuring proteins, due to their effect on ice microstructure. The interaction between the proteins and ice is based on an intimate surface-surface complementarity given by a spatial matching 
between the distance of the amino acids of the polypeptide chain and the lattice spacing of the crystal. The equilibrium configuration of AFPs attached to ice results from an entropy-driven optimization process which minimizes the Gibbs free energy.

Two concepts are generally used when modeling thermal hysteresis due to AFPs. On the one hand there is the nucleation growth described by the Gibbs-Thomson equation (Kaptay 2011) and on the other hand a nonlinear adsorption kinetics of the ice-binding proteins. Li \& Luo (1993) used the first approach. They proposed a model for the thermal hysteresis activity based on the first order Gibbs-Thomson relation using hard sphere theory by Reiss et al. (1959) and compared the results with experimental data of the winter flounder (Psodeupleuronectes americanus) antifreeze protein type I (HPLC6). An example for the second approach is given by Liu \& $\mathrm{Li}$ (2006). They applied a two-dimensional reversible adsorption kinetic model for thermal hysteresis activity on a hyperactive antifreeze protein of the insect Tenebrio molitor. Also the pinning or "stones on a pillow" model developed by Sander \& Tkachenko (2004) assumed an adsorption kinetics and can therefore be counted among the second school.

In the following we use a novel approach to justify the thermal hysteresis due to AFPs and apply Landau's phase transition theory. The two model approaches mentioned above describe a non-colligative behavior of the freezing point depression as a function of AFP-concentration, but do not always reflect the experimental values adequately. The well-established Landau theory of first order phase transitions can be used to describes changes of the Gibbs free energy during the transition between the ordinary hexagonal ice I modification and the supercooled liquid. Therefore, a Landau model is best suited to describe the thermal hysteresis caused by AFPs. In a recent work (Kutschan et al. 2014) we applied Landau's phase transition theory to study the dynamical aspects of the nucleation growth and calculated the critical size of the crystallization seed as a function of the AFP concentration. Here we use Landau's theory to describe the impact of AFPs on the lower limit of the supercooling region and calculate the AFP concentration with the maximal possible freezing point depression.

The outline of the paper is as follows. In the next section we introduce the Landau's approach to phase transitions, according to which a single variable, the order parameter $\psi$, is introduced to keep track of the phase change. The phase transition is modified due to AFPs, which we describe by a second variable, the AFP-concentration $\mathrm{Q}$. The description of the experimental conditions and the determination of the model parameters are presented in the second and third section, leading to the Landau free energy describing the thermal hysteresis due to the AFPs of Fragilariopsis cylindrus. The calculation of the AFP concentration with the maximal possible freezing point depression completes the fourth section. In the fifths section we summarize and conclude. 


\section{Landau theory of phase transition}

\section{General aspects}

A crucial point in Landau's theory plays the order parameter due to his duality of meanings. An order parameter $\psi$ bridges the gap between a microscopic point of view and a macroscopic perspective (Errington \& Debenedetti 2001, Medvedev \& Naberukhin 1987) and describes a measure of the degree of order of a system (Fig. 2). Such a first order phase transition is associated with a symmetry change during the transition from liquid water to hexagonal ice in the classification of the point groups. It is assumed that the free energy density $\varphi$ can be described by a power series expansion (Brokate \& Sprekels 1996, Kittel \& Krömer 2001). For a first order phase transition our approach enables a double welled function whose two minima correspond to two stable phases (Harrowell \& Oxtoby 1987). We couple the order parameter with $\widetilde{\psi}$ the function of the AFPs $\beta_{1}(\rho)$ and obtain the Gibbs free energy density $\varphi$

$$
\begin{aligned}
\varphi\left(\widetilde{\psi}^{j}\right) & =K\left(\beta_{0}\left(\beta_{1}, \beta_{2}, \beta_{3}\right), \beta_{1}, \beta_{2}, \beta_{3}\right) \\
& +\left(\beta_{1}-\frac{1}{12} \beta_{2} \beta_{3}+\frac{1}{216} \beta_{3}^{3}\right) \widetilde{\psi}^{j}+\left(\frac{1}{2} \beta_{2}-\frac{1}{24} \beta_{3}^{2}\right)\left(\widetilde{\psi}^{j}\right)^{2}+\left(\widetilde{\psi}^{j}\right)^{4}
\end{aligned}
$$

with

$$
\begin{aligned}
K\left(\beta_{0}, \beta_{1}, \beta_{2}, \beta_{3}\right) & =\left(\widetilde{\psi}^{j}\right){ }_{1}^{2} \widetilde{\psi}_{2}^{j} \widetilde{\psi}_{3}^{j} \\
& =\frac{1}{864}\left(-12 \beta_{2}+\beta_{3}^{2}\right)^{2} \cos ^{2}\left(\frac{1}{3} \arccos (\delta)+\frac{2 \pi}{3}\right) \\
& \cdot\left(-1+2 \cos ^{2}\left(\frac{1}{3} \arccos (\delta)+\frac{2 \pi}{3}\right)\right)
\end{aligned}
$$

and

$$
\delta=-\frac{216 \beta_{1}-18 \beta_{2} \beta_{3}+\beta_{3}^{3}}{\left(-12 \beta_{2}+\beta_{3}^{2}\right)^{\frac{3}{2}}}
$$

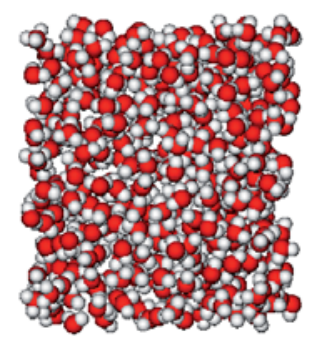

Fig. 2. $\widetilde{\psi}_{0}$

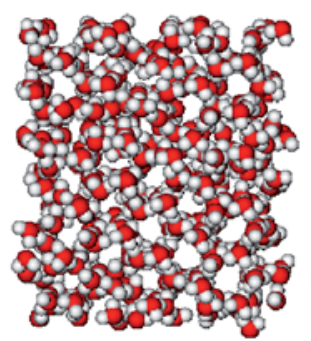

$\widetilde{\psi}_{1}$

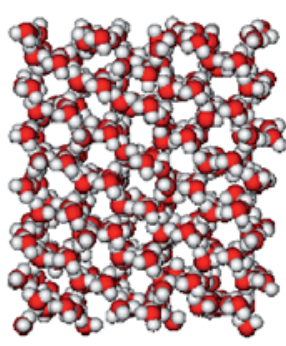

$$
\widetilde{\psi}_{2}
$$

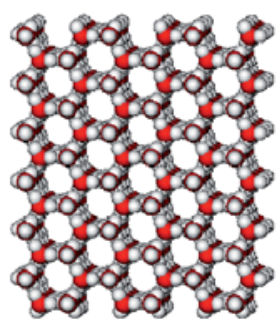

$<\widetilde{\psi}_{3}$ degree of low order (Brownian random process) $\rightarrow$ degree of high order. 
derived in Appendix Eq. (33). The index $j$ modifies only the constant $K$ in the potential function (1) as shown in Fig. 9 in Appendix. We choose $j=1$ because this case describes the supercooling process.

However, the case $j=0$ characterizes the superheating process and the case $j=2$ connects both branches. The coefficient $\beta_{2}=-\mathrm{p} / C_{1}$ corresponds to the negative dimensionless AFP-concentration, whereby $\rho$ designated the AFP-concentration which becomes dimensionless by the constant $C_{1}$. Furthermore, $\beta_{2}$ is a function of the temperature and vanishes for the critical temperature $T=T_{c}$. The quadratic expression $\frac{1}{2} \beta_{2} \psi^{2}$ in Eq. (19) change its sign at this point due

$\beta_{2}=B\left(T-T_{c}\right)$.

Figure 3 shows the asymmetric potential with a saddle point for $\rho=0$ and $T=T_{c}$. On the other hand we have a temperature $T=T_{2}$ that coincides with the actual first-order transition temperature that corresponds to a symmetric potential (Figs. 3 and 4), i. e. the linear term in Eq. (1) have to vanish

$\beta_{1}-\frac{1}{12} \beta_{2}^{T_{2}} \beta_{3}+\frac{1}{216} \beta_{3}^{3}=0$

and one obtains $B=\left(12 \frac{\beta_{1}}{\beta_{3}}+\frac{1}{18} \beta_{3}^{2}\right) /\left(T_{2}-T_{c}\right)$ and for $\beta_{2}$

$\beta_{2}=\left(12 \frac{\beta_{1}}{\beta_{3}}+\frac{1}{18} \beta_{3}^{2}\right) \frac{T-T_{c}}{T_{2}-T_{c}}$.

Finally, $\beta_{2}$ should only be dependent on the temperature $T$ but not on the (negative) AFP concentration $\beta_{1}$ and set $\beta_{1}=0$

$\beta_{2}(T)=\frac{1}{18} \beta_{3}^{2} \frac{T-T_{c}}{T_{2}-T_{c}}$

with the special value $\beta_{2}\left(T_{2}\right)=\beta_{2}^{\mathrm{T}_{2}}=\frac{1}{18} \beta_{3}^{2}$. The reason for this is, that we understand $\beta_{2}$ as state variable dependent on the temperature only, whereas $\beta_{1}(\rho)$ describes an external field dependent on the AFPs.

Now, we consider the symmetric potential for $\beta_{1}=0$ according to Eq. (34) in Appendix

$\varphi(\widetilde{\psi})=\frac{1}{20736} \beta_{3}^{4}+\left(\frac{1}{2} \beta_{2}-\frac{1}{24} \beta_{3}^{2}\right) \widetilde{\psi}^{2}+\widetilde{\psi}^{4}$

and determine the extreme values $\frac{d \varphi(\widetilde{\psi})}{d \widetilde{\psi}}=\widetilde{\psi}\left(\beta_{2}-\frac{1}{12} \beta_{3}^{2}+4 \widetilde{\psi}^{2}\right)=0$. Therefore we find the maximum for $\widetilde{\psi}_{c}^{0}$ and the both minima 
74 Kutschan, Thoms \& Bayer-Giraldi

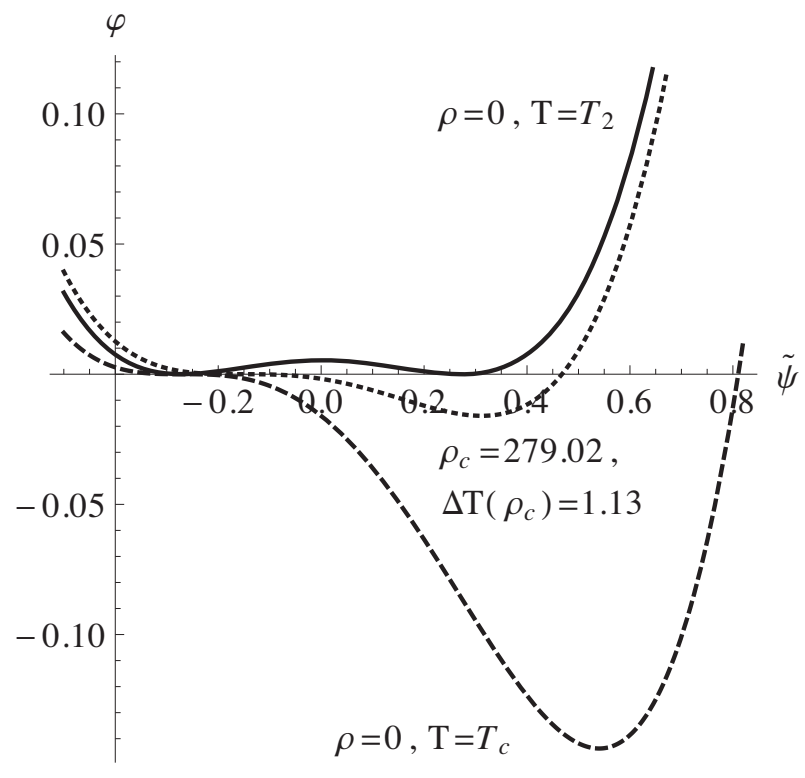

Fig. 3. The free energy $\varphi$ versus the order parameter $\psi$ for pure water $(\rho=0$, phase transition at $T=T_{2}$, temperature at maximal supercooling $T_{\mathrm{c}}=233.15 \mathrm{~K}$ ) and for the saturation concentration of AFPs $\rho=\rho_{c}$.

Fig. 4. Dependence of the absolute minimum of the free energy (9) on temperature $T$ for $\rho=0$. The step at phase transition $\left(T=T_{2}\right)$ indicates the phase change if one jumps from the first minimum to the absolute one in Figure 3.

$\widetilde{\psi}_{c}= \pm \frac{\beta_{3}}{4 \sqrt{3}} \sqrt{1-\frac{2}{3} \frac{T-T_{c}}{T_{2}-T_{c}}}$

The parameter $\beta_{3} \neq 0$ is essential for a first order phase transition and determines the jump at $T=T_{2}$. The coefficient $\beta_{3}$ characterizes the jump for a first-order phase transition at the freezing point temperature $T=T_{2}$ [see Eq. (7) and Fig. 4]. 


\section{Experimental conditions}

Fragilariopsis cylindrus shows a moderate thermal hysteresis activity (Bayer-Giraldi et al.2011). The thermal hysteresis experiments were performed at low salinity of $S=$ $10 \mathrm{~g} / \mathrm{kg}$ using a recombinant protein (Bayer-Giraldi et al. 2011) with a broad concentration range. The actual physiological concentration is unknown, since no measurements have been done in sea ice. The freezing point depression $\Delta T_{f}$ on the basis of the salinity can be calculated from $\Delta T_{f}=K_{f} S / M_{\mathrm{NaCl}}$ with the cryoscopic constant $K_{f}=$ $1.853 \mathrm{Kkg} / \mathrm{mol}$ for water and the molar mass for sodium chloride $M_{\mathrm{NaCl}}=58.45 \mathrm{~g} / \mathrm{mol}$ and obtain $\Delta T_{f}=0.63 \mathrm{~K}$. Hence, one finds the freezing temperature for the salinity $T_{2}=T_{m}-\Delta T_{f}=272.52 \mathrm{~K}$ for $S=10$ with $T_{m}=273.15 \mathrm{~K}$.

\section{Parameter identification}

From the potential (1) it can be found a nonlinear relation (44) for the temperature depression $\Delta T$ due to AFP concentration $\rho$

$$
\Delta T+1=-\frac{3}{4 \beta_{3}^{2}} \sqrt{\frac{-3456 \frac{\rho}{c_{1}} \beta_{3} \delta^{2}+\beta_{3}^{4}\left(9-8 \delta^{2}\right)}{\delta^{4}}} \cos \left(\frac{1}{3} \arccos (\varepsilon)+\frac{2 \pi}{3}\right)+\frac{9}{8 \delta^{2}}-\frac{3}{2}
$$

with

$$
\varepsilon=\frac{1492992 \beta_{1}^{2} \delta^{4}-1728 \beta_{1} \beta_{3}^{3} \delta^{2}\left(-9+4 \delta^{2}\right)+\beta_{3}^{6}\left(27-36 \delta^{2}+8 \delta^{4}\right)}{\beta_{3} \delta^{2}\left(-3456 \beta_{1} \delta^{2}+\beta_{3}^{3}\left(-9+8 \delta^{2}\right)\right) \sqrt{\frac{1}{\delta^{4}}\left(3456 \beta_{1} \beta_{3} \delta^{2}+\beta_{3}^{4}\left(9-8 \delta^{2}\right)\right)}}
$$

A nonlinear parameter fitting method provides always $\delta=1$ for our measured values, so that Eq. (10) is simplified to

$$
\Delta T+1=-\frac{3}{4 \beta_{3}^{2}} \sqrt{-3456 \frac{\rho}{C_{1}} \beta_{3}+\beta_{3}^{4}} \cos \left(\frac{1}{3} \arccos (\varepsilon)+\frac{2 \pi}{3}\right)-\frac{3}{8}
$$

with

$$
\varepsilon=\frac{-1492992\left(-\frac{\rho}{C_{1}}\right)^{2}+8640 \frac{\rho}{C_{1}} \beta_{3}^{3}+\beta_{3}^{6}}{\left(-3456 \frac{\rho}{C_{1}} \beta_{3}+\beta_{3}^{4}\right)^{\frac{3}{2}}}
$$

according to Eq. (45) in Appendix. There are two unknown values in equation (12), the parameter $\beta_{3}$ and a scaling factor $C_{1}$ for the AFP concentration. These parameters are determined for Fragilariopsis cylindrus using a nonlinear estimation method as 


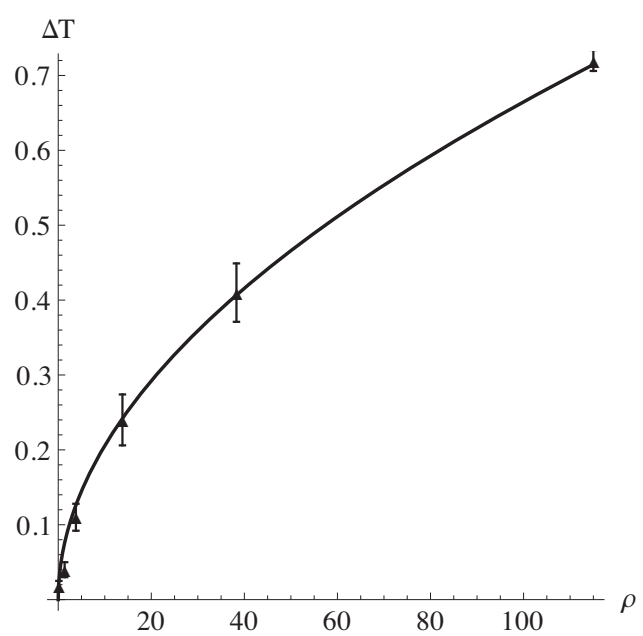

\begin{tabular}{l|l|l}
\hline$\rho$ in $\mu \mathrm{M}$ & $\Delta T$ & $s_{\Delta T}$ \\
\hline 0.138 & 0.018 & 0.007 \\
1.38 & 0.04 & 0.010 \\
3.8 & 0.11 & 0.018 \\
13.8 & 0.24 & 0.034 \\
38.3 & 0.41 & 0.039 \\
115 & 0.72 & 0.014 \\
\hline
\end{tabular}

Fig. 5. Parameter identification for Fragilariopsis cylindrus (26 kDa) according to Eq. (12): $C_{1}$ $=9193.38, \beta_{3}=-3.2416$.

shown in Figure 5. The model reflects the measured values except for a constant shift conditioned by $\Delta T+1 \sim \Delta T$. The Gibbs free energy density (1) can be expressed as

$\varphi(\widetilde{\psi})=\mathrm{K}\left(\beta_{1}, \beta_{3}\right)+\beta_{1} \widetilde{\psi}-\frac{\beta_{3}^{2}}{72} \widetilde{\psi}^{2}+\widetilde{\psi}^{4}$

for $\beta_{2}=\frac{1}{18} \beta_{3}^{2}$ with the translation $K\left(\beta_{1}, \beta_{3}\right)$

$$
\begin{aligned}
& K\left(\beta_{1}, \beta_{3}\right)=\frac{\beta_{3}^{4}}{7776} \cos ^{2}\left(\frac{1}{3} \arccos \left(-\frac{648 \sqrt{3} \beta_{1}}{\left(\beta_{3}^{2}\right)^{\frac{3}{2}}}\right)+\frac{2 \pi}{3}\right) \\
& \cdot\left(-1+2 \cos ^{2}\left(\frac{1}{3} \arccos \left(-\frac{648 \sqrt{3} \beta_{1}}{\left(\beta_{3}^{2}\right)^{\frac{3}{2}}}\right)+\frac{2 \pi}{3}\right)\right)
\end{aligned}
$$

An absolute potential cannot be determined but only the difference between two potentials. An additional condition such as a double root in Eq. (20) can be used in order to determine this arbitrary constant $K\left(\beta_{1}, \beta_{3}\right)$. If one chooses $K=0$

$$
\varphi(\widetilde{\psi}, K=0)=\beta_{1} \widetilde{\psi}-\frac{\beta_{3}^{2}}{72} \widetilde{\psi}^{2}+\widetilde{\psi}^{4}
$$

one sees the shift based on $K$ between $\varphi\left(K\left(\beta_{1}, \beta_{3}\right)\right)$ and $\varphi(K=0)$ (compare Fig. 6). 

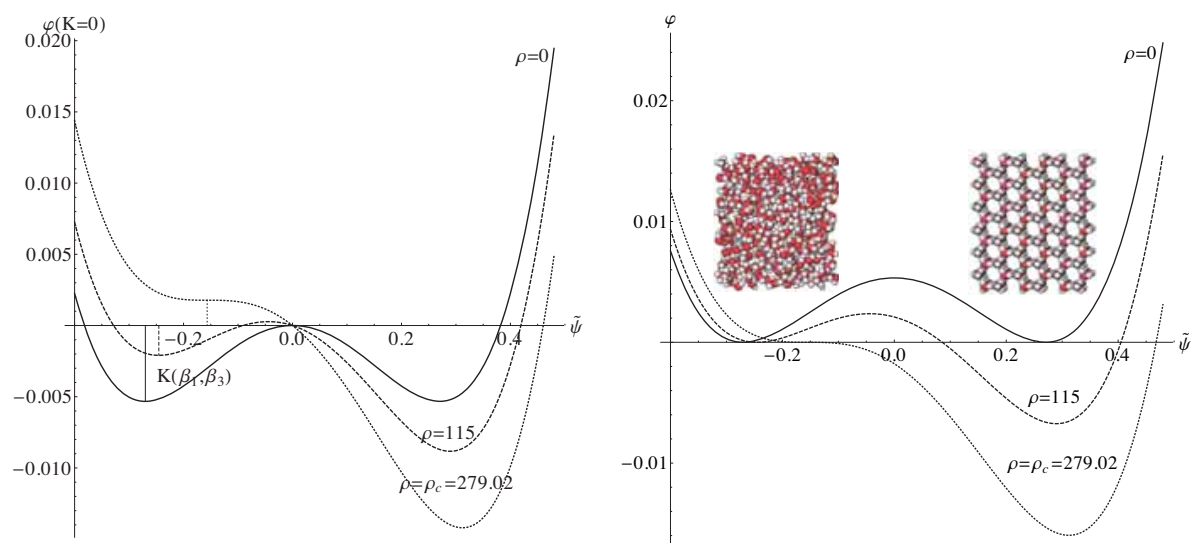

Fig. 6. The free energy $\varphi$ versus the order parameter $\widetilde{\psi}$ for various AFP concentrations $\rho$ at constant temperature $T=T_{2}$ (left: $\varphi$ for the arbitrary constant $K=0$, right: $\varphi$ renormalized to zero at the first minimum). In the right figure, the absolute minimum indicates the contribution of the AFPs to $\varphi$.

\section{Limit of maximal AFP concentration}

The extreme values of the potential $\varphi(20)$ or (1) are determined by $\frac{d \varphi(\widetilde{\psi})}{d \widetilde{\psi}}=0$ and yields

$\widetilde{\psi}^{3}+\left(\frac{1}{4} \beta_{2}-\frac{1}{48} \beta_{3}^{2}\right) \widetilde{\psi}+\frac{1}{4} \beta_{1}-\frac{1}{48} \beta_{2} \beta_{3}+\frac{1}{864} \beta_{3}^{3}=0$

if we identify $\widetilde{\psi}$ with $\widetilde{\psi}_{1}$ in Eq. (20). The index " 1 " can be omitted because the index of the multiple root $\widetilde{\psi}_{1}$ of multiplicity 2 is chosen arbitrarily. With $\beta_{2}=\frac{1}{18} \beta_{3}^{2}$ and $\beta_{1}=-\frac{\rho}{C_{1}}$ one obtains the loops of a bistable system

$\rho=C_{1}\left(4 \widetilde{\psi}^{3}-\frac{1}{36} \beta_{3}^{2} \tilde{\psi}\right)$

or rather by means of Eq. (27)

$$
\widetilde{\psi}^{j}=\frac{\sqrt{\beta_{3}^{2}}}{6 \sqrt{3}} \cos \left(\frac{1}{3} \arccos \left(\frac{648 \sqrt{3} \rho}{C_{1}\left(\beta_{3}^{2}\right)^{\frac{3}{2}}}\right)+\frac{2 \pi}{3} j\right)
$$

with $j=0,1,2$ (see Fig. 7 resp. Fig. 8). The function (16) has a maximum and a minimum $\widetilde{\psi}^{ \pm}= \pm \widetilde{\psi}_{c}= \pm \frac{1}{12 \sqrt{3}} \sqrt{\beta_{3}^{2}}$ deducible from the condition $\frac{d \rho}{d \widetilde{\psi}}=0$. The extreme values decompose the profile into two stable branches for $\widetilde{\psi}<-\widetilde{\psi}_{c}$ or $\widetilde{\psi}>-\widetilde{\psi}_{c}$ and 
an unstable region for $-\widetilde{\psi}_{c}<\widetilde{\psi}<\widetilde{\psi}_{c}$ characterized by the dotted line in Figure 7 . The appropriate values of the function are $\rho\left( \pm \widetilde{\psi}_{c}\right)= \pm \rho_{c}= \pm \frac{C_{1}}{648 \sqrt{3}}\left(\beta_{3}^{2}\right)^{\frac{3}{2}}$. Besides $\widetilde{\psi}\left(-\rho_{c}\right)=\widetilde{\psi}_{c}=\frac{1}{12 \sqrt{3}} \sqrt{\beta_{3}^{2}}$ there is $\tilde{\psi}\left(\rho_{c}\right)=\widetilde{\psi}_{f}=\frac{1}{6 \sqrt{3}} \sqrt{\beta_{3}^{2}}$ for the branch $\tilde{\psi}^{j=0}$ (Fig. 8). According to the phase jump in Eq. (9) for $T=T_{2}$ in Fig. 4 one find

$$
\tilde{\psi}^{j}(\rho=0)=\left\{\begin{array}{rrr}
\frac{1}{12} \beta_{3}=-\tilde{\psi}_{\mathrm{r}} & \text { for } & j=0 \\
0 & \text { for } & j=2 \\
-\frac{1}{12} \beta_{3}=\tilde{\psi}_{\mathrm{r}} & \text { for } & j=1
\end{array}\right.
$$

for a pure water/ice system without AFPs. The dashed lines in Figures 7 and 8 correspond to the measured loop for the AFP concentration of $115 \mu \mathrm{M}$ and the lines to the concentration of $\rho_{\mathrm{c}}=279.02 \mu \mathrm{M}$ that induce a maximal freezing point depression of $\Delta T=1.13 \mathrm{~K}$ as a maximal theoretic limit. Accordingly, the minima of the energy density change their shape and position, depending on the AFP concentration at the freezing temperature $T_{2}$ in Figure 6 . The minima are much smaller than the minimum for the supercooling temperature $T_{\mathrm{c}}$ of pure water without AFPs in Figure 3 . The line of the value $\rho_{\mathrm{c}}$ describes precisely the limit for the inflection points. The limit lies not so far away from the measured values (Bayer-Giraldi et al. 2011). AFP-concentrations up to $350 \mu \mathrm{M}$ was measured and seems to converge to a saturation value, $\rho=275 \mu \mathrm{M}$ corresponding to delta $\Delta T=0.91 \mathrm{~K}$.

The instability region of the loop shown in Figure 7 can also be understood as Maxwell construction. The line at $\rho=0$ shown in Figure 8 disconnects the supercooled and the superheated region. It should be mentioned here that both Maxwell's phase transition theory of real gases and Landau's phase transition theory base on a polynomial approach.

\section{Summary}

Until now we considered an isotropic order parameter. This simplification was sufficient in order to derive a suitable non colligative relation between temperature depression and AFP-concentration. The Landau approach can reproduce the experimental values except for a constant shift. Our function is more complex than a simple power law for the AFP concentration as suggested by thermal hysteresis experiments. However, with the parameter set derived for Fragilariopsis cylindrus the modelled curve is comparable with the often used square root law (Raymond \& DeVries 1977). Moreover, we determined a theoretical limit for the maximal effective AFP concentration. It is recommended to confirm our theoretical limit by means of appropriate measurements. It is useful to include an anisotropic order parameter with the aim to investigate the change of the symmetry elements of the point group of the ice crystal. 


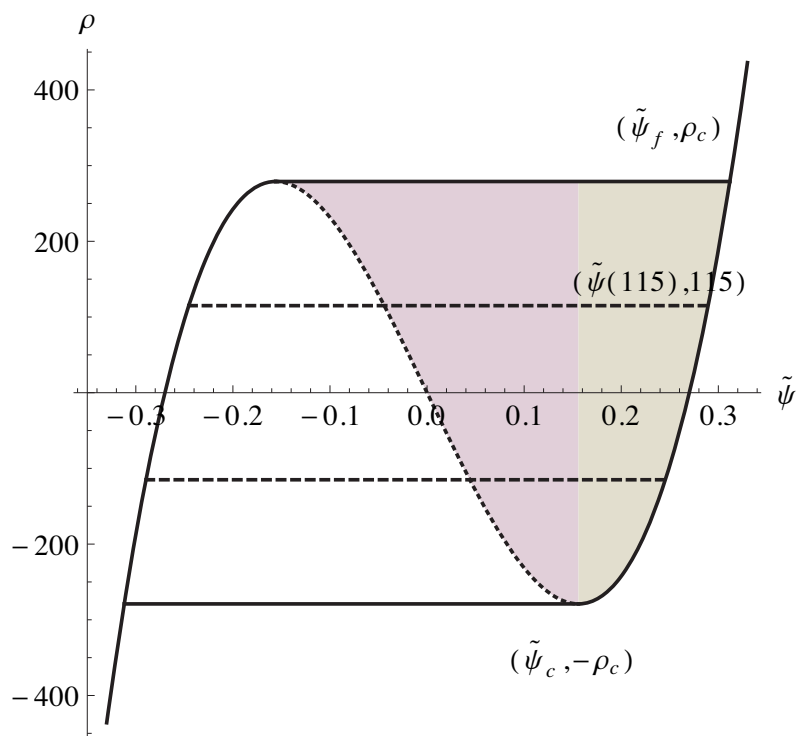

Fig. 7. Relationship between the AFP concentration $\rho$ and the two minima of the free energy $\varphi$ at $T=T_{2}$ (solid lines). The maximal AFP concentration $\rho_{c}$ is defined by the limiting inflection point in Figure 6.

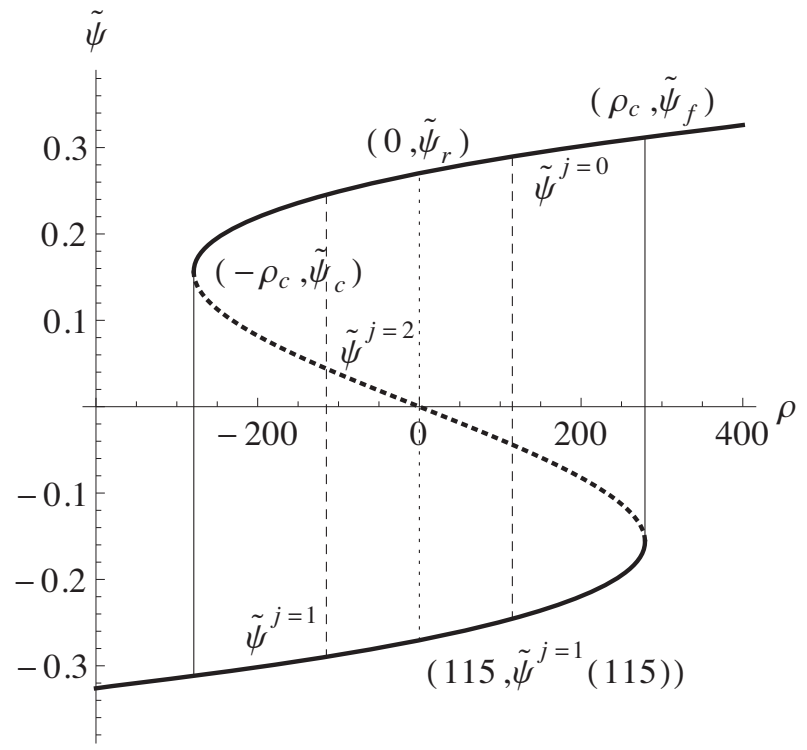

Fig. 8. The inverse of the function in Figure 7. Here, the maximal AFP concentration $\rho_{c}$ is defined by the termination of the solution branch for the first minimum in the Figure 6 . 


\section{Acknowledgements}

This work is based on the results of the DFG Priority Program SPP 1158 "Antarctic Research".

\section{Appendix}

The potential $\varphi$ can be expressed by a polynomial

$$
\varphi(\psi)=\beta_{0}+\beta_{1} \psi+\frac{1}{2} \beta_{2} \psi^{2}+\frac{1}{3} \beta_{3} \psi^{3}+\frac{1}{4} \beta_{4} \psi^{4}
$$

with $\beta_{1}=\alpha_{1}-\rho$. The shift $\psi=\widetilde{\psi}-\frac{1}{3} \frac{\beta_{3}}{\beta_{4}}$ yields

$$
\begin{aligned}
\varphi(\widetilde{\psi}) & =\beta_{0}-\frac{1}{12} \beta_{1} \beta_{3}+\frac{1}{288} \beta_{2} \beta_{3}^{2}-\frac{1}{6912} \beta_{3}^{4} \\
& +\left(\beta_{1}-\frac{1}{12} \beta_{2} \beta_{3}+\frac{1}{216} \beta_{3}^{3}\right) \widetilde{\psi}+\left(\frac{1}{2} \beta_{2}-\frac{1}{24} \beta_{3}^{2}\right) \widetilde{\psi}^{2}+\widetilde{\psi}^{4} \\
& =\left(\widetilde{\psi}-\widetilde{\psi}_{1}\right)^{2}\left(\widetilde{\psi}-\widetilde{\psi}_{2}\right)\left(\widetilde{\psi}-\widetilde{\psi}_{3}\right)
\end{aligned}
$$

with $\beta_{4}=4$. The requirement $\widetilde{\psi}_{1}=\widetilde{\psi}_{4}$ for the factorization using the fundamental theorem of algebra in Eq. (20) $\varphi(\widetilde{\psi})=\left(\widetilde{\psi}-\widetilde{\psi}_{1}\right)\left(\widetilde{\psi}-\widetilde{\psi}_{2}\right)\left(\widetilde{\psi}-\widetilde{\psi}_{3}\right)\left(\widetilde{\psi}-\widetilde{\psi}_{4}\right)=$ $\left(\widetilde{\psi}-\widetilde{\psi}_{1}\right)^{2}\left(\widetilde{\psi}-\widetilde{\psi}_{2}\right)\left(\widetilde{\psi}-\widetilde{\psi}_{3}\right)$ reduces the number of independent coefficients from four down to three. The method of equating the coefficients allows us to find the factors

$$
\begin{aligned}
\beta_{0}-\frac{1}{12} \beta_{1} \beta_{3}+\frac{1}{288} \beta_{2} \beta_{3}^{2}-\frac{1}{6912} \beta_{3}^{4} & =\widetilde{\psi}_{1}^{2} \widetilde{\psi}_{2} \widetilde{\psi}_{3} \\
\beta_{1}-\frac{1}{12} \beta_{2} \beta_{3}+\frac{1}{216} \beta_{3}^{3} & =-\widetilde{\psi}_{1}\left(\widetilde{\psi}_{1} \widetilde{\psi}_{3}+2 \widetilde{\psi}_{2} \widetilde{\psi}_{3}+\widetilde{\psi}_{1} \widetilde{\psi}_{2}\right) \\
\frac{1}{2} \beta_{2}-\frac{1}{24} \beta_{3}^{2} & =\widetilde{\psi}_{1}^{2}+2 \widetilde{\psi}_{1}\left(\widetilde{\psi}_{2}+\widetilde{\psi}_{3}\right)+\widetilde{\psi}_{2} \widetilde{\psi}_{3} \\
0 & =-\left(2 \widetilde{\psi}_{1}+\widetilde{\psi}_{2}+\widetilde{\psi}_{3}\right) \\
1 & =1 .
\end{aligned}
$$

The requirement $\widetilde{\psi}_{1}=\widetilde{\psi}_{4}$ yields to a double root and the four dimensional parameter space $\left\{\beta_{0}, \beta_{1}, \beta_{2}, \beta_{3}\right\}$ reduces to a three dimensional parameter space $\left\{\beta_{1}, \beta_{2}, \beta_{3}\right\}$ with $\beta_{0}=\beta_{0}\left(\beta_{1}, \beta_{2}, \beta_{3}\right)$ according to Eq. (21). The substitution of $\widetilde{\psi}_{2}$ and $\widetilde{\psi}_{3}$ in the system of equation (22) to (24) yields

$$
\widetilde{\psi}_{1}^{3}+\left(\frac{1}{4} \beta_{2}-\frac{1}{48} \beta_{3}^{2}\right) \widetilde{\psi}_{1}+\frac{1}{4} \beta_{1}-\frac{1}{48} \beta_{2} \beta_{3}+\frac{1}{864} \beta_{3}^{3}=0
$$


with the real solutions

$\widetilde{\psi}_{1}=\frac{1}{6} \sqrt{-12 \beta_{2}+\beta_{3}^{2}} \cos \left(\frac{1}{3} \arccos (\delta)+\frac{2 \pi}{3} j\right)$

for $j=0,1,2$ and

$\delta=-\frac{216 \beta_{1}-18 \beta_{2} \beta_{3}+\beta_{3}^{3}}{\left(-12 \beta_{2}+\beta_{3}^{2}\right)^{\frac{3}{2}}}$

for the casus irreducibilis. We consider the solution for $j=0,1,2$

$\widetilde{\psi}_{1}^{j}=\frac{1}{6} \sqrt{-12 \beta_{2}+\beta_{\frac{2}{3}}} \cos \left(\frac{1}{3} \arccos (\delta)+j \frac{2 \pi}{3}\right)$

and obtain the other zeros in consideration of (23) and (24)

$\widetilde{\psi}_{2}^{j}=-\widetilde{\psi}_{1}^{j} \pm \sqrt{-2\left(\tilde{\psi}_{1}^{j}\right)^{2}-\frac{1}{2} \beta_{2}+\frac{1}{24} \beta_{\frac{2}{3}}}$

and

$\widetilde{\psi}_{3}^{j}=-\widetilde{\psi}_{1}^{j} \mp \sqrt{-2\left(\widetilde{\psi}_{1}^{j}\right)^{2}-\frac{1}{2} \beta_{2}+\frac{1}{24} \beta_{3}^{2}}$

Thus, the constant in the potential (20) is

$$
\begin{aligned}
\mathrm{K} & =\left(\widetilde{\psi}_{1}^{j}\right)^{2} \widetilde{\psi}_{2}^{j} \widetilde{\psi}_{3}^{j}=\beta_{0}-\frac{1}{12} \beta_{1} \beta_{3}+\frac{1}{288} \beta_{2} \beta_{3}^{2}-\frac{1}{6912} \beta_{3}^{4} \\
& =\frac{1}{864}\left(-12 \beta_{2}+\beta_{3}^{2}\right)^{2} \cos ^{2}\left(\frac{1}{3} \arccos (\delta)+j \frac{2 \pi}{3}\right) \\
& \cdot\left(-1+2 \cos ^{2}\left(\frac{1}{3} \arccos (\delta)+j \frac{2 \pi}{3}\right)\right)
\end{aligned}
$$

and the potential function

$$
\begin{aligned}
\varphi\left(\tilde{\psi}^{j}\right) & =\frac{1}{864}\left(-12 \beta_{2}+\beta_{3}^{2}\right)^{2} \cos ^{2}\left(\frac{1}{3} \arccos (\delta)+j \frac{2 \pi}{3}\right) \\
& \cdot\left(-1+2 \cos ^{2}\left(\frac{1}{3} \arccos (\delta)+j \frac{2 \pi}{3}\right)\right) \\
& +\left(\beta_{1}-\frac{1}{12} \beta_{2} \beta_{3}+\frac{1}{216} \beta_{3}^{3}\right) \tilde{\psi}^{j} \\
& +\left(\frac{1}{2} \beta_{2}-\frac{1}{24} \beta_{3}^{2}\right)\left(\tilde{\psi}^{j}\right)^{2}+\left(\tilde{\psi}^{j}\right)^{4} .
\end{aligned}
$$



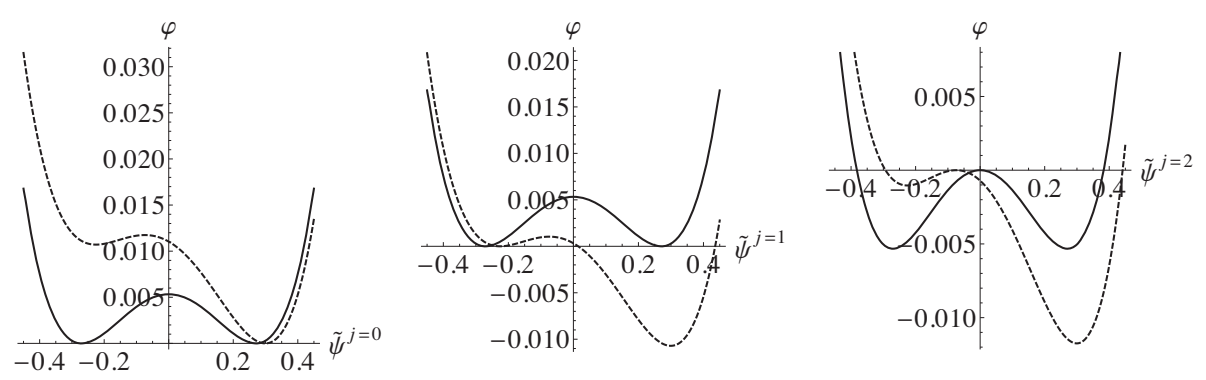

Fig. 9. Potential $\varphi\left(\widetilde{\psi}^{j}\right)$ Eq. (33) for $j=0,1,2$ with $\beta_{3}=-3.2416, \beta_{2}=\frac{1}{18} \beta_{3}^{2}, \beta_{1}=0$ (straight line) and $\beta_{1}=-0.02$ (dashed line). The potential is symmetric if $\beta_{1}-\frac{1}{12} \beta_{2} \beta_{3}+\frac{1}{216} \beta_{3}^{3}=0 \Rightarrow \beta_{2}=\frac{1}{18} \beta_{3}^{2}+12 \frac{\beta_{1}}{\beta_{3}}$ and the
equation (33) is simplified to

$\varphi\left(\widetilde{\psi}^{j}\right)=\frac{\left(-432 \beta_{1}+\beta_{3}^{3}-144 \beta_{3}\left(\widetilde{\psi}^{j}\right)^{2}\right)^{2}}{20736 \beta_{3}^{2}}$

for $j=0,1$ and

$\varphi\left(\widetilde{\psi}^{j}\right)=\left(6 \frac{\beta_{1}}{\beta_{3}}-\frac{1}{72} \beta_{3}^{2}\right)\left(\tilde{\psi}^{j}\right)^{2}+\left(\widetilde{\psi}^{j}\right)^{4}$

for $j=2$. The potential (20) depends on the zeros (27) and on $\delta$ accordingly.

Therefore we solve the equation

$-\left(-12 \beta_{2}+\beta_{3}^{2}\right)^{\frac{3}{2}} \delta=216 \beta_{1}-18 \beta_{2} \beta_{3}+\beta_{3}^{3}$.

We obtain

$$
\begin{aligned}
\beta_{2}^{3} & +\left(\frac{3}{16} \frac{\beta_{3}^{2}}{\delta^{2}}-\frac{1}{4} \beta_{3}^{2}\right) \beta_{2}^{2}+\left(-\frac{9}{2} \frac{\beta_{1} \beta_{3}}{\delta^{2}}-\frac{1}{48} \frac{\beta_{3}^{4}}{\delta^{2}}+\frac{1}{48} \beta_{3}^{4}\right) \beta_{2} \\
& +27 \frac{\beta_{1}^{2}}{\delta^{2}}+\frac{1}{4} \frac{\beta_{1} \beta_{3}^{3}}{\delta^{2}}+\frac{1}{1728} \frac{\beta_{3}^{6}}{\delta^{2}}-\frac{1}{1728} \beta_{3}^{6}=0
\end{aligned}
$$

move $\beta_{2}=\widetilde{\beta}_{2}-\frac{1}{3}\left(\frac{3}{16} \frac{\beta_{3}^{2}}{\delta^{2}}-\frac{1}{4} \beta_{3}^{2}\right)$ and get 


$$
\begin{aligned}
\widetilde{\beta}_{2}^{3} & +\left(-\frac{3}{256} \frac{\beta_{3}^{4}}{\delta^{4}}-\frac{9}{2} \frac{\beta_{1} \beta_{3}}{\delta^{2}}+\frac{1}{96} \frac{\beta_{3}^{4}}{\delta^{2}}\right) \widetilde{\beta}_{2} \\
& +\frac{1}{2048} \frac{\beta_{3}^{6}}{\delta^{6}}+\frac{9}{32} \frac{\beta_{1} \beta_{3}^{3}}{\delta^{4}}-\frac{1}{1536} \frac{\beta_{3}^{6}}{\delta^{4}}+27 \frac{\beta_{1}^{2}}{\delta^{2}}-\frac{1}{8} \frac{\beta_{1} \beta_{3}^{3}}{\delta^{2}}+\frac{1}{6912} \frac{\beta_{3}^{6}}{\delta^{2}}=0 .
\end{aligned}
$$

The solution is

$\widetilde{\beta}_{2}=\frac{1}{24} \sqrt{\frac{3456 \beta_{1} \beta_{3} \delta^{2}+\beta_{3}^{4}\left(9-8 \delta^{2}\right)}{\delta^{4}}}\left(\cos \frac{1}{3} \arccos (\varepsilon)+j \frac{2 \pi}{3}\right)$

resp.

$\beta_{2}=\frac{1}{24} \sqrt{\frac{3456 \beta_{1} \beta_{3} \delta^{2}+\beta_{3}^{4}\left(9-8 \delta^{2}\right)}{\delta^{4}}} \cos \left(\frac{1}{3} \arccos (\varepsilon)+j \frac{2 \pi}{3}\right)-\frac{1}{16} \frac{\beta_{3}^{2}}{\delta^{2}}+\frac{1}{12} \beta_{3}^{2}$

with $\varepsilon=\frac{1492992 \beta_{1}^{2} \delta^{4}-1728 \beta_{1} \beta_{3}^{3} \delta^{2}\left(-9+4 \delta^{2}\right)+\beta_{3}^{6}\left(27-36 \delta^{2}+8 \delta^{4}\right)}{\beta_{3} \delta^{2}\left(-3456 \beta_{1} \delta^{2}+\beta_{3}^{3}\left(-9+8 \delta^{2}\right)\right) \sqrt{\frac{1}{\delta^{4}}\left(3456 \beta_{1} \beta_{2} \delta^{2}+\beta_{3}^{4}\left(9-8 \delta^{2}\right)\right)}}$

for $j=0,1,2$. The coefficient $\beta_{2}(T)$ implies the measured temperature $T$, the critical temperature $T_{\mathrm{c}}$, and the actual freezing temperature $T_{2}$

$$
\begin{aligned}
\beta_{2} & =\beta_{2}+\beta_{2}=\frac{1}{18} \beta_{3}^{2} \frac{T-T_{c}}{T_{2}-T_{c}}=\frac{1}{18} \beta_{3}^{2} \frac{T-T_{2}+T_{2}-T_{c}}{T_{2}-T_{c}} \\
& =\frac{1}{18} \beta_{3}^{2}\left(\frac{T-T_{2}}{T_{2}-T_{\mathrm{c}}}+1\right)=\frac{1}{18} \beta_{3}^{2}\left(\frac{\Delta T}{T_{2}-T_{\mathrm{c}}}+1\right)
\end{aligned}
$$

with $\beta_{2}^{\prime}=\frac{1}{18} \beta_{3}^{2} \frac{\Delta T}{T_{2}-T_{c}}$ and $\beta_{2}^{\prime}=\frac{1}{18} \beta_{3}^{2}$. Combining the equations (40) and (41) we get

$$
\begin{aligned}
\frac{\Delta T}{T_{2}-T_{C}}+1 & =\frac{3}{4 \beta_{3}^{2}} \sqrt{\frac{-3456 \frac{\rho}{C_{1}} \beta_{3} \delta^{2}+\beta_{3}^{4}\left(9-8 \delta^{2}\right)}{\delta^{4}}} \cos \left(\frac{1}{3} \arccos (\varepsilon)+j \frac{2 \pi}{3}\right) \\
& -\frac{9}{8 \delta^{2}}+\frac{3}{2}
\end{aligned}
$$

for $\beta_{1}=-\rho / C_{1}$. The reflection across the $\mathrm{x}$-axis and the dimensionless scaling factor $C_{2}=\frac{T_{2}-T_{c}}{1 K}$ yields 


$$
\begin{aligned}
C_{2} \frac{\Delta T}{T_{2}-T_{C}}+1 & =-\frac{3}{4 \beta_{3}^{2}} \sqrt{\frac{-3456 \frac{\rho}{C_{1}} \beta_{3} \delta^{2}+\beta_{3}^{4}\left(9-8 \delta^{2}\right)}{\delta^{4}}} \\
& \cdot \cos \left(\frac{1}{3} \arccos (\varepsilon)+j \frac{2 \pi}{3}\right)+\frac{9}{8 \delta^{2}}-\frac{3}{2}
\end{aligned}
$$

or for $j=1$

$\Delta T+1=-\frac{3}{4 \beta_{3}^{2}} \sqrt{\frac{-3456 \frac{\rho}{C_{1}} \beta_{3} \delta^{2}+\beta_{3}^{4}\left(9-8 \delta^{2}\right)}{\delta^{4}}} \cos \left(\frac{1}{3} \arccos (\varepsilon)+\frac{2 \pi}{3}\right)+\frac{9}{8 \delta^{2}}-\frac{3}{2}$.

The maximal supercooling is obtained for $\delta=1$. For this case equation (44) is simplified to

$$
\begin{aligned}
& \Delta T+1=-\frac{3}{4 \beta_{3}^{2}} \sqrt{-3456 \frac{\rho}{C_{1}} \beta_{3}+\beta_{3}^{4}} \cos \left(\frac{1}{3} \arccos (\varepsilon)+\frac{2 \pi}{3}\right)-\frac{3}{8} \\
& \text { with } \varepsilon=\frac{-1492992\left(-\frac{\rho}{C_{1}}\right)^{2}+8640 \frac{\rho}{C_{1}} \beta^{3}+\beta_{3}^{6}}{\left(-3456 \frac{\rho}{C_{1}} \beta_{3}+\beta_{3}^{4}\right)^{\frac{3}{2}}}
\end{aligned}
$$

\section{References}

Barrett, J. (2001): Thermal hysteresis proteins. - IJBCB 33: 105-117.

Bartsch, A. (1989): Die Eisalgenflora des Weddelmeeres (Antarktis): Artenzusammensetzung und Biomasse sowie Ökophysiologie ausgewählter Arten (Sea ice algae of the Weddel Sea (Antartica): Species composition, biomass, and ecophysiology of selected species). - Ber. Polarforschung 63: $110 \mathrm{p}$.

Bayer-Giraldi, M., Weikusat, I., Besir, H. \& Dieckmann, G. (2011): Characterization of an antifreeze protein from the polar diatom Fragilariopsis cylindrus and its relevance in sea ice. - Cryobiology 63: 210-219.

Bayer-Giraldi, M., Uhlig, C., John, U., Mock, T. \& Valentin, K. (2010): Antifreeze proteins in polar sea ice diatoms: diversity and gene expression in the genus fragilariopsis. - Environmental Microbiology 12: 1041-1052.

Brokate, M. \& Sprekels, J. (1996): Hysteresis and Phase Transitions. - Springer-Verlag, New York, Berlin, Heidelberg, $357 \mathrm{p}$.

Brown, M. \& Bowman, J. (2001): A molecular phylogenetic survey of sea-ice microbial communities (SIMCO). - FEMS, Microbiology, Ecology 35: 267-275.

Errington, J. \& Debenedetti, P. (2001): Relationship between structural order and the anomalies of liquid water. - Nature 409: 318-321.

Günther, S. \& Dieckmann, S. (2001): Vertical zonation and community transition of sea-ice diatoms in fast ice and platelet layer, Weddell Sea, Antarctica. - Annals of Glaciology 33: 287-296. 
Harrowell, P. \& Oxtoby, D. (1987): On the interaction between order and a moving interface: Dynamical disordering and anisotropic growth rates. - The Journal of Chemical Physics 86: 29322942.

Kaptay, G. (2011): The Gibbs equation versus the Kelvin and the Gibbs-Thomson equations to describe nucleation and equilibrium of nano-materials. - Journal of Nanoscience and Nanotechnology 12: 1-9.

Kittel, C. \& Krömer, H. (2001): Thermodynamik. Oldenbourg-Verlag, München, Wien, 463 p.

Kristiansen, E. \& Zachariassen, K. (2005): The mechanism by which fish antifreeze proteins cause thermal hysteresis. - Cryobiology 51: 262-280.

Kutschan, B., Morawetz, K. \& Thoms. S. (2014): Dynamical mechanism of antifreeze proteins to prevent ice growth. - Phys. Rev. E. 90: 022711.

Li, Q. \& Luo, L. (1993): The kinetic theory of thermal hysteresis of a macromolecule solution. Chem. Phys. Lett. 216: 453-457.

Liu, J. \& Li, Q. (2006): Theoretical model of antifreeze protein - ice adsorption: Binding of large ligands to a two-dimensional homogeneous lattice. - Chem. Phys. Lett. 422: 67-71.

Lizotte, M. (2001): The Contributions of Sea Ice Algae to Antarctic Marine Primary Production. American Zoologist 41: 57-73.

Medvedev, N. \& Naberukhin, Y. (1987): Shape of the delaunay simplices in dense random packings of hard and soft spheres. - J. Non-Cryst. Solids 94: 402-406.

Raymond, J. \& DeVries, A. (1977): Adsorption inhibition as a mechanism of freezing resistance in polar fishes. - Physiological Sciences 74: 2589-2593.

Reiss, H., Frisch, H. \& Lebowitz, J. (1959): Statistical mechanics of rigidspheres. - The Journal of Chemical Physics 31: 369-380.

Roberts, K., Granum, E., Leegood, R. \& Raven, J. (2007): Carbon acquisition by diatoms. - Photosynth. Res. 93: 79-88.

Sander, L.O. \& Tkachenko, A. (2004): Kinetic pinning and biological antifreezes. - Phys. Rev. Lett. 93: $128102-1-128102-4$.

Thomas, D. \& Dieckmann G. (2002): Antartic sea ice - a habitat for extremophiles. - Science 295 : 641-644.

Manuscript received September, 28, 2015, accepted May, 23, 2016 